\title{
Dongting Lake Floodwater Diversion and Storage Modeling and Control Architecture Based on the Next Generation Network
}

\author{
Lianqing Xue, Zhenchun Hao, Dan Li, and Xiaoqun Liu \\ State Key Laboratory of Hydrology-Water Resources and Hydraulic Engineering, \\ Hohai University, Nanjing, 210098,P.R. China \\ lqxue@hhu.edu.cn
}

\begin{abstract}
Dongting Lake is one of the most important floodwater diversion and storage regions in Yangzi River basin. The development of the next generation network and information technology makes it possible to implement the remote monitoring and floodwater control effectively. By taking advantage of the next generation network and lots of 16-byte addresses of IPv6, different kinds of remote sensing devices can be accessed and monitored data can be collected and transferred to the two-dimension hydraulic model for Dongting Lake. And on the basis of real-time network data transmission and parallel algorithm the model can be operated with more unstructured meshes and the hydrological regime and gates are virtually controlled by remote sensors. When analyzing the modeling results, the input data and computing results can be revised in time according to the feedback from the high-speed internet-based system and the project operation can be effectively managed as well.
\end{abstract}

Keywords: the Next-Generation Network, Network Application, Parallel Computation Algorithm, Hydraulic Model.

\section{Introduction}

At present, Yangzi River flood control and its hydrological information management are the most important issues in hydrology study field. Because of its size and complicated geographic features, Dongting Lake region has become the most important flood diversion route and water storage area after water diversion from Sikou, on the south bank of Jingjiang River, to Dongting Lake. The efficiency of flood control and regulation command dispatching, however, has been greatly lowered by the previous single motor running and manual data input, and can not satisfy the requirement of hydraulic management using information technology.. Therefore, it is important to utilize the new generation network with overall regulation for real-time monitoring and operation to model the floodwater diversion and storage in Dongting Lake region using high-speed parallel computing method; to set up a platform for water information monitoring and fast transmission; to enhance flood forecast and control; and to use high-tech for flood control regulation, decision-making, evaluation and management after flood disaster. The transition from flood control to flood management will 
play a critical role in flood control of the middle and lower reaches of the Yangzi River. It is also important for the economy development of the Yangzi River basin.

The current problems in floodwater diversion and storage of Dongting Lake region are as follows. (1) Dongting Lake region covers a large area and has a large amount gates. It is difficult to carry out real-time monitoring and information transmission. Therefore, a high-speed information transmission platform should be set to transfer the collected data timely to the model for decision-making; (2) the involved regions are very complicated. The related theory model for floodwater diversion and storage can not be simulated exactly. It needs continuous feedback and revision for the regulation results. The internet-based floodwater diversion and storage management system for Dongting Lake region has the functions of remote data acquisition, monitoring alarm, simulation, real-time operation and revision by the feedback. Next generation network and the standard TCP/IP protocol are essential because of the requirement of video images transmission and varieties of sensors and data.

The system architecture design mainly involves two aspects: (1) real-time data acquisition; and (2) simulation. Firstly, it is simulated according to the real-time data. Secondly, the remote gates are operated based on the simulation results; and at the same time, the real-time data is obtained for subsequent monitoring and modification. The internet-based floodwater diversion and storage control system will be discussed below, including the simulation algorithm and the results.

\section{Monitoring and Control System Based on Internet}

By using a lot of 16-byte addresses of IPv6, different kinds of sensing devices are accessed and the monitoring data for each diversion area such as environment or earthquake monitoring data can be collected in time, which shows the "more powerful and more convenient "characteristic of next generation network.

Fig.1. shows the floodwater diversion and storage control system for Dongting Lake region. It mainly consists of two parts: (1) a local area network composed of sensors and controllers. The collected data is transmitted by a gateway through internet to the center. There are different kinds of sensors in the system used for monitoring and control. They are water level sensor, flow velocity sensor, water flow direction sensor, etc. Another kind of data is the video image. The real-time information can be transmitted through high-speed network to the center by the pickup cameras that are laid on some important places, on the gates for instance. In order to operate the gates successfully, some remote controllers are needed. Then each component needs an IP address for overall control. For enough address space, Ipv6 can be set in each component; (2) the center, composed of high-speed computers with parallel computing capacity. The control center makes simulation according to the real time data, controls the controllers based on the simulation results, and thereafter achieves the modification and recycling monitoring.

Each aspect should be considered during the system architecture design, such as communication, sensor, simulation, and control. The network and data control, monitoring, regulation, simulation, feedback and web browser should also be included in this system. The TCP/IP and web technology make it possible for the system be 


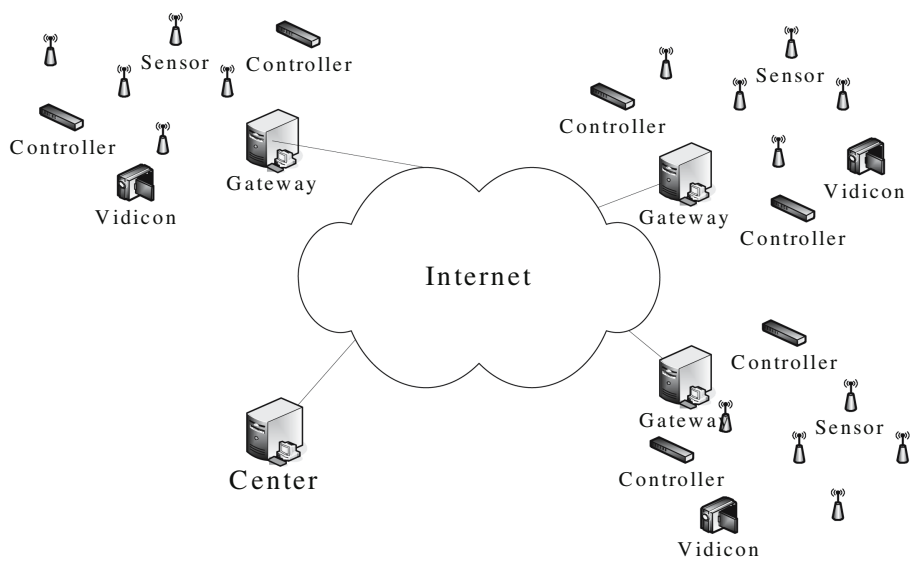

Fig. 1. General Architecture of the internet-based system

effectively controlled at any time and at any place. The internet-based system should include the functions of combined control, simulation, data collection and analysis, management.

There are two essential differences between this system and those traditional internetbased systems: (1) more simulation computing; (2) real-time information transmission.

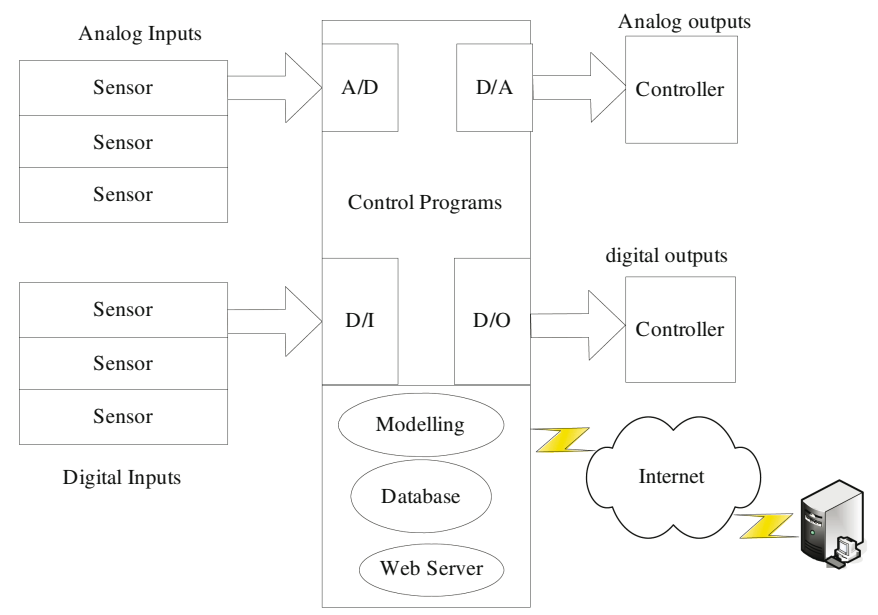

Fig. 2. the Internet-based system for floodwater diversion and storage modeling

Fig.2. shows the architecture of the internet-based remote system for floodwater control and management. Most of them are same as that of traditional ones, such as transmission protocol, safety control, sensor and database. The major difference is that there is a special modeling layer in the internet-based architecture for data simulation, which will be discussed in next section. 


\section{Two-Dimension Hydraulic Model for Flood Routing}

Dongting Lake region covers more than $5000 \mathrm{~km}^{2}$ with 24 diversion areas. Therefore, it is very complicated to simulate this region. The internet-based remote control system introduced above is constructed to solve this problem. This section will mainly discuss the simulation of the model. The model is verified by the historical records. The results show that next generation network and high-speed parallel computers make it possible to do remote real-time control for the Dongting Lake region. The following will discuss the theoretic model and give some examples.

\subsection{Hydraulic Model Theory}

With the development and application of computer technology, study on adjusting the capacity of Dongting Lake is based on mature hydraulic theory. A two-dimension model is set up to simulate the flood routing of Dongting Lake region and its diversion areas, and to quantify the flood storage and detention capacity of the wetland. As FVM can be directly used in irregular region, it's employed with unstructured mesh for area partition here. In the paper, the two-dimension shallow water equation is transformed from conservative form to discrete form. The two-dimension shallow water equation is set up by selecting water level and flow as controlling variables.

(1) two-dimension hydraulic equation

$$
\begin{gathered}
\frac{\partial h}{\partial t}+\frac{\partial(h u)}{\partial x}+\frac{\partial(h v)}{\partial y}=0 \\
\frac{\partial(h u)}{\partial t}+\frac{\partial\left(h u^{2}+g h^{2} / 2\right)}{\partial x}+\frac{\partial(h u v)}{\partial y}=g h\left(s_{0 x}-s_{f x}\right) \\
\frac{\partial(h v)}{\partial t}+\frac{\partial(h u v)}{\partial x}+\frac{\partial\left(h v^{2}+g h^{2} / 2\right)}{\partial y}=g h\left(S_{0 y}-S_{f y}\right)
\end{gathered}
$$

Where, $\mathrm{h}$ is water depth; $\mathrm{u}$ and $\mathrm{v}$ are vertical mean velocity along $\mathrm{x}$ and $\mathrm{y}$ direction separately; $S_{0 x}$ and $S_{f x}, S_{0 y}$ and $S_{f y}$ are lake bed slope and friction slope along $x$ and y direction separately; $g$ is gravity acceleration.

Set $\vec{q}=\{h, h u, h v\}^{T}$, a state column vector composed of variables that represent flow conservation. set $\vec{f}(q)=\left\{h u, h u^{2}+g h^{2} / 2, h u v\right\}^{T}$ as the flux vector along x direction, and $\vec{g}(q)=\left\{h u, h u v, h v^{2}+g h^{2} / 2\right\}^{T}$ as the flux vector along y direction. When only the bed slope and friction are considered, the source and sink term $\vec{b}(q)$ can be written as follows:

$$
\frac{\partial \vec{q}}{\partial t}+\frac{\partial \vec{f}(q)}{\partial x}+\frac{\partial \vec{g}(q)}{\partial y}=\vec{b}(q)
$$


(2) Discretization principle of FVM

The explicit form is adopted for space discretization of the continuous equation, and the leapfrog method for time discretization. Crossing grid is used for physical variables calculation. The input or output flow and momentum flux along normal in each discrete unit boundary are worked out. By water and momentum balance equation, the averaged water depth and velocity at the end time are gained at last (under the assumption of averagely distributed hydraulic variables). The details of discretization will not be discussed in this paper.

According to the above theory, the procedures of FVM model are: First, derive water depth $h_{L}$, flow velocity $u_{L}, v_{L}$ and wave velocity $C_{L}$ of the calculated unit grid from those in earlier period. Second, derive those of the adjacent grid as well as normal flux $f_{L R}(\cdot)$ by the known water depth and flow velocity. Repeat step 2 for other units next to the already calculated ones. Finally, calculate the water depth h, flow velocity $\mathrm{u}$ and $\mathrm{v}$ in current period.

\subsection{Parallel Computing of Flood Routing}

In order to improve the efficiency of flood routing modeling, it is necessary to selectively accept and renew real-time monitoring information, and to take advantage of the parallel computing on internet. Recently, the domain decomposition method has gained more and more attentions from people and it is becoming an important branch of the parallel computing science. If the common grids of adjacent areas can be reduced during region partition, the time of calculation will be much shorter with higher efficiency by using the high-speed network transmission. Since the key problem of parallel procedure design is task allocation and communication, in this paper, Message Passing Interface (MPI) is adopted as the information transmission database, which performed well in communication.

\subsection{Grid Generation}

On the basis of the above theory, the corresponding procedures are programmed. The mesh is generated using GIS to digitize the 1:10000 groundwater measured data in 1995. Then the flood regulation in Dongting Lake region is simulated, which are the theoretical basis for flood control in Yangzi River basin.

The measured data of the east Dongting Lake, south Dongting Lake and Muping Lake in 1995 are collected. Program interface is developed based on GIS. The quadrilateral mesh for hydraulic calculation is generated by the grid topology program. The principle of grid arrangement is to adopt as few grids to represent the trend of topography as possible, and to make sure that they are reasonably organized and gradually varied. In order to reduce the modeling time, it is essential to adopt different grid scale. Considering the complicated condition in Dongting Lake region, different grid density is used, which not only guarantee the simulation of the flood diversion capacity of Caowei River in the north, but also reflect flood routing characteristics of the south lake as well as the general distribution of topography. The smallest grid is $60 \mathrm{~m} * 62 \mathrm{~m}$, and there are grids up to 100,664 in the south lake area. Fig.3. shows the grid arrangement. 


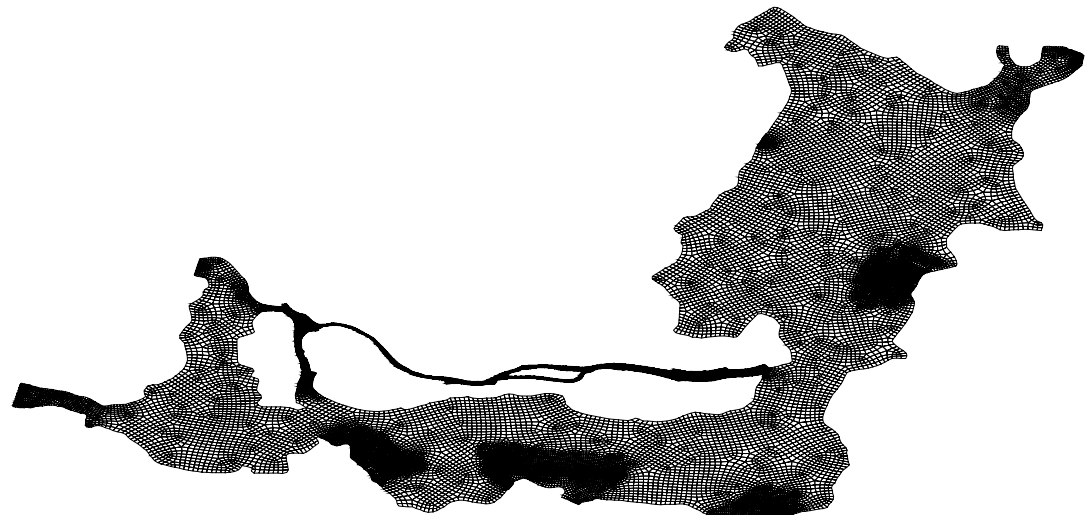

Fig. 3. Calculation Grids of Dongting Lake

\subsection{Simulation Analysis}

In this paper, the flood routing from 0:00 am, July $21^{\text {th }}$ to $23: 00 \mathrm{pm}$, August $2^{\text {th }}$ in typical year 1998, is simulated and compared with the parallel computing results. The results are in good agreement with measured flood routing trend. The model gives a reliable simulation with the average water level discrepancy of $0.11 \mathrm{~m}$. Therefore, it can be used for quantificational prediction of flood diversion process.

While there are 24 diversion areas in Dongting Lake region, only one mode of gate operation is listed in this paper. The results show that if the gates were opened, the total flood storage volume of five diversion areas would be $64.6^{*} 10^{8} \mathrm{~m}^{3}$. The five diversion areas are Gongshuangcha, Qiannianghu, Datonghu Jianshe and Jianxin. For the flood in 1998, lake area met the floodwater diversion and storage request from July $21^{\text {th }}$ till August $2^{\text {th }}$, and the volume of storage water was $54 * 10^{8} \mathrm{~m}^{3}$. Water level of outside lake dropped $0.3 \mathrm{~m}$. However, it became uniform with natural water level

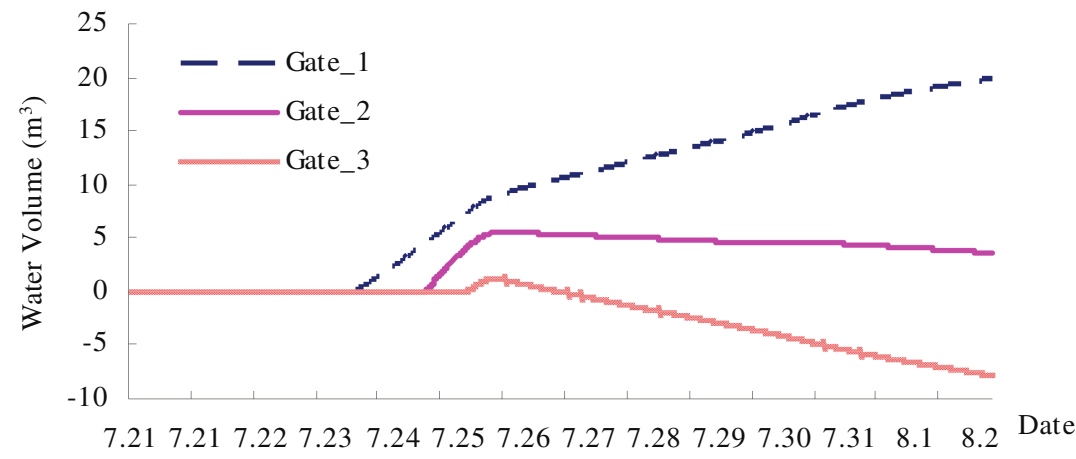

Fig. 4. Water inflow and outflow hydrograph for gate1, gate2 and gate $3\left(10^{8} \mathrm{~m}^{3}\right)$ 


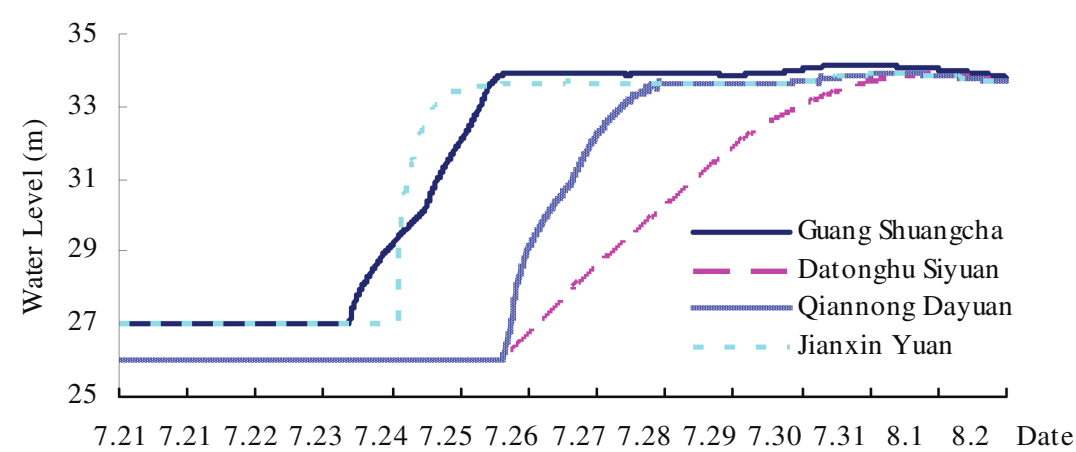

Fig. 5. Water level hydrograph in 4 different places

since August 2nd. The diversion areas lost their floodwater diversion and storage function. Gate No. 1 in Gongshuangcha lies in upstream, where the relative water level is higher. So it was opened earlier than Gate No. 2 and Gate No. 3. It can be observed from Fig.4. Gate No. 1 was opened all the time, while Gate No. 2 was opened after Gate No. 1. The volume of passed flow in Gate No. 1 and Gate No. 2 are positive. Gate No. 3 was closed in the beginning and opened later. The volume of passed flow changed from positive to negative. The above Results show that it is inadequate to use diversion areas in Dongting Lake region. Therefore, it is very important to adopt highspeed parallel computing method. Fig.5. shows the water level hydrograph.

\section{Conclusions}

By taking advantage of new generation network, floodwater diversion and storage control system for Dongting Lake region is constructed and discussed in this paper. Satisfying application results shows that the system is proficient in real-time data acquisition, high-speed information transmission, and remote control, which can effectively improve the efficiency of flood control and regulation command dispatching. Meanwhile, comparing the internet-based system for Dongting Lake with the traditional systems, we find that the main difference is the use of high-speed parallel computing for simulation in the internet-based system. Furthermore, the twodimension hydraulic model for Dongting Lake employs Finite Volume Method (FVM) and unstructured mesh for area partition. The flood routing in a typical year is modeled and the regulation effects of 24 diversion areas are predicted, during which the hydrological regime and gates are virtually controlled by remote sensors and controllers. It is also proved that the application of new generation network can be fully broadened in future because it's more powerful and more convenient. Moreover, it is of great practical value in future flood control.

Acknowledgments. This paper has been funded by the National Natural Science Foundation of China under Grant No. 50609006, the Key Project of Chinese Ministry of Education under Grant No. 105084, the Natural Science Foundation of Jiangsu Province under Grant No. BK2006092, and the Excellent Youth Teacher of Southeast University Program under Grant No. 4009001018. 


\section{References}

1. Paul I-Hai Lin, Harold L. Broberg, Internet-Based Monitoring and Controls for HVAC Applications, IEEE Industry Applications Magazine, (Jan-Feb 2002), 49-54

2. Paul I-Hai Lin, Web Programming and Applications. Fort Wayne: Indiana UniversityPurdue University, (2001).

3. Chen Jin, Huang Wei, Inquiry into system risk analytic method of flood control engineering. Journal of Yangtze River Scientific Research Institute, (2001) 18(5): 37-40.

4. Piotr Małoszewski, Przemysław Wachniew and Piotr Czupryński, Study of hydraulic parameters in heterogeneous gravel beds: Constructed wetland in Nowa Słupia (Poland). Journal of Hydrology, Volume 331, Issues 3-4, 15 December (2006) 630-642

5. Zhang Yanzhao, Dong Jie, Han Min. Research of flood routing simulation system based on GIS. Chinese Journal of Scientific Instrument, (2006)27(6): 936-937.

6. Yu Yunli, Wang Deguan, Wang Zhigang, Lai Xijun, Numerical Simulation of Thermal Discharge Based on FVM Method, Journal of Ocean University of China. (2006) 5(1): 7-11

7. Minhyung Kim, Sangkyun Kim and Arcy J. Kong, High performance AAA architecture for massive IPv4 networks. Future Generation Computer Systems, Volume 23, Issue 2, (February 2007) 275-279.

8. A.Bejan and S.Lorente, Constructal tree-shaped flow architecture. Applied Thermal Engineering, Volume 27, Issue 4, (March 2007) 755-761 